\title{
Magnetophoretic separation of diamagnetic particles through parallel
}

\author{
ferrofluid streams
}

\author{
Ahmed Munaz, ${ }^{a}$ Muhammad J. A. Shiddiky, ${ }^{\text {a,b }}$ and Nam-Trung Nguyen, ${ }^{\text {a }}$ \\ ${ }^{a}$ Queensland Micro- and Nanotechnology Centre, Griffith University, Brisbane, QLD 4111, \\ Australia
}

*Email: nam-trung.nguyen@griffith.edu.au

${ }^{b}$ School of Natural Sciences, Griffith University, Nathan Campus, QLD 4111, Australia

Highlights

- Separation of nonmagnetic particles with a magnetic field.

- Parallel streams of ferrofluid with different concentrations to minimize accumulation of magnetic nanoparticles.

- Separation of diamagnetic particles with small size difference.

- Three-stream Flow-focusing configuration results in more efficient separation

Abstract: Particle separation based on microfluidic technology offers a simple, reliable, and low-cost approach for the diagnosis of diseases. The separation concept can be extended to genetic engineering, cell transplantation, and immunology. This paper reports a simple microfluidic platform for the separation of diamagnetic particles of different sizes utilizing parallel ferrofluid streams. The ferrofluid streams with predefined concentrations of magnetic nanoparticles promote negative magnetophoresis and are able to separate a particle mixture with a subtle size variation. Numerical simulation was used to optimise the magnetic field gradient, e.g. the number and position of the external permanent magnets. The effect of flow rate ratio and the concentration distribution were analyzed by the simulation and validated by experiments. Furthermore, two-stream and three-stream ferrofluid configurations were evaluated to find the optimum separation performance. The experimental results show a 
maximum separation efficiency of $78 \%$ and $75 \%$ with three-stream configuration for $3.2-\mu \mathrm{m}$ and $4.8-\mu \mathrm{m}$ particles, respectively.

Keywords: magnetofluidics; negative magnetophoresis; particle separation; ferrofluid

\section{Introduction:}

Negative magnetophoresis is the migration of non-magnetic particles in a magnetic fluid and a magnetic field [1]. Negative magnetophoresis has been attracting considerable attention from the industry and the research community, as the phenomenon can be used for sample preparation steps such as separation and concentration of cells on a microfluidic platform [2, 3]. Negative magnetophoresis finds applications in a range of areas such as disease diagnostics, environmental monitoring, therapeutics study, and single cell analysis [4]. Negative magnetophoresis can separate diamagnetic particles such as cells in a paramagnetic medium such as a solution of ferrofluid. Manipulation of diamagnetic cells is enabled through an applied external magnetic field $[5,6]$. Cells and other diamagnetic particles experience a magnetic force that attracts them toward the low magnetic field potential. Particle separation based on negative magnetophoresis has its unique advantages as well as challenges. Negative magnetophoresis offers contactless, label-free manipulation that reduces the processing time of an assay and lowers the associated cost. Moreover, this technique is non-invasive and does not induce heat into the sample [6]. The challenges of negative magnetophoresis are the biocompatibility of the magnetic fluid for long-term investigations, the attachment of magnetic nanoparticles to cells, and the required washing steps for subsequent off-chip investigations. Despite these challenges, improvements have been reported recently. Zhao et al. reported the rapid separation of HeLa cells and red blood cells based on the difference in their size [7]. The customized ferrofluid retains cell viability for up to two hours. In another work, the same team demonstrated the enrichment of rare circulating tumour cells (CTCs) from undiluted white 
blood cells (WBCs) using negative magnetophoresis with a recovery rate of $92.9 \%$ and a purity of $11.7 \%$ [8]. The debris from the blood sample was filtered and focused by the sheath flow. The applied magnetic field separated the large CTCs along with some of the large WBCs from smaller WBCs. The collected cells had an excellent proliferation capability, short-term viability, and clear expression of biomarkers.

Based on their size, cells can be deflected into two streams due to the mismatch in magnetisation that uses a function of cell volume. The deflection can further be tuned by optimizing other physical properties such as flow rate, viscosity, and size of the nanoparticles in the ferrofluid [9]. Shih et al. showed the effect of flow rate and magnetic field strength on the separation of bacteria bound to magnetic nanoparticles [10]. Optimising these parameters offers a separation efficiency of up to $90 \%$. Pamme et al. demonstrated the continuous separation of mouse macrophages and tumor cells tagged with magnetic nanoparticles [11]. The separation depends on the flow rates, the size of the cells, magnetic loading, and the magnetic moment of the external magnets. Efficient separation also depends on the geometry, the orientation of magnetisation, size, and distance of the magnets from the microchannel [12]. Xia et al. utilised structures such as microcombs and microneedles to focus the magnetic field to a strong gradient inside a microchannel [13]. Efficient separation with high-throughput was possible for red blood cells and E.coli cells tagged with magnetic nanoparticles. Wilbanks et al. demonstrated the effects of magnet alignment that can increase the rate of particle trapping in a ferrofluid flow [14]. Gourikutty et al. reported the isolation of CTCs from whole blood sample without tagging with tumor-specific antigen [15]. First, a range of customised permanent magnet was used to isolate white blood cells from whole blood with a capture efficiency of up to $99.9 \%$. Next, an optimised micro slit membrane depleted red blood cells, keeping only the target tumor cells. Liang et al. utilised negative magnetophoresis through a U-shape microchannel to focus and separate $5-\mu \mathrm{m}$ and $15-\mu \mathrm{m}$ polystyrene particles suspended 
in a diluted ferrofluid [16]. The U-shape geometry offered sheath-free focusing at the inlet and efficient separation at the outlet of the microchannel. Yan et al. used sheathless magnetophoresis to separate $6-\mu \mathrm{m}$ magnetic and $13-\mu \mathrm{m}$ polystyrene particles through a groovebased microchannel. The design focused the magnetic particles at the centre, whereas the nonmagnetic particles moved along the side wall of the microchannel [17].

Biological cells usually have size varying from one micrometre to several micrometres. For instance, the volume of HeLa cells and red blood cells are on the order of $3700 \pm 1500 \mu \mathrm{m}^{3}$ and $66 \pm 8.3 \mu \mathrm{m}^{3}$, respectively $[18,19]$. The diameters of WBCs and CTCs may vary between 8 to14 $\mu \mathrm{m}$, and 15 to $25 \mu \mathrm{m}$ respectively [8]. As smaller CTCs may also exist in the blood circulation [20], cancer diagnosis techniques relying on the isolation of CTCs from a clinical sample are vulnerable due to the similar size and density of CTCs and WBCs [21]. The size of the CTCs may depend on the cancer population, thus the separation performance may vary significantly [22]. Therefore, separating clinically relevant rare cells with a subtle variation in size is technically challenging. Moreover, difficult tasks such as separation of homogeneous and heterogeneous cells can provide useful insights in cell synchronization, counting of dead cells and early-stage disease diagnosis [23, 24]. Most of the previous works focused on particle separation with a relatively large size difference. For instance, Liang et al. showed the separation of $2.88-\mu \mathrm{m}$ magnetic and $10-\mu \mathrm{m}$ diamagnetic particles in a ferrofluid solution in a T-shaped microchannel [25]. Zhu et al. demonstrated the separation of 1- $\mu \mathrm{m}, 1.9-\mu \mathrm{m}$, and 3.1$\mu \mathrm{m}$ from $9.9-\mu \mathrm{m}$ diamagnetic particle inside of a ferrofluid flow using a simple permanent magnet [26]. Due to the magnetic buoyancy force, large particles were deflected further than the smaller ones.

The separation of small particles is crucial for various applications. Pathogenic bacteria, and infected cells often have a size difference of less than $5 \mu \mathrm{m}$. Rapid sorting of these bacteria and infected cells could potentially allow for the detection of food poisoning and blood 
infections [22]. Thus highly sensitive and efficient platforms are sought after by the industry and the scientific community. Very few works reported the use of magnetophoresis to separate particles with diameters of less than $5 \mu \mathrm{m}$. The main challenge here is the small difference in magnetisation due to small size difference that makes the separation very difficult. As the difference is less in these samples, a slight change in physical parameters such as flow rate, magnetization of the magnet and the volume fraction of the magnetic nanoparticles in the ferrofluid could significantly affect the separation performance [9]. Most works on small-scale particle separation were based on inertial focusing with curved or spiral microchannels [27, 28]. As separation depends on the inertial force of the channel geometry, new devices need to be developed [22].

Recent works on magnetophoretic separation of particles with a subtle variation in size have been reported. For an instance, Zhou et al. explored the shape-based separation of particles with the same volume. The spherical and peanut shaped particles (fusion of two spherical particles) resulted in a different magnetic and drag force, thus can be separated in a ferrofluid flow. Zhu et al. reported the separation of magnetic and non-magnetic particles based on their magnetic properties in a solution of commercial ferrofluid [29]. The 4.2- $\mu \mathrm{m}$ and 7.3$\mu \mathrm{m}$ non-magnetic particles were separated from the $2.6-\mu \mathrm{m}$ and $7.9-\mu \mathrm{m}$ magnetic particles, respectively. Successful separation of $2.8-\mu \mathrm{m}$ and $8.2-\mu \mathrm{m}$ magnetic particles were also reported.

Permanent magnets arranged with opposite polarities across a microchannel creates cell trapping regions. Magnets with same polarities focus the cells into a single stream. Hejazian et al. demonstrated the concentration process of two nonmagnetic particles based on their size difference [30]. Magnet arrays with opposite polarity created a number of trapping zones along the channel. The large nonmagnetic particles were trapped between the two attractive magnets in the regions of field minima. The smaller nonmagnetic particles were trapped in the region 
of field maxima near the magnetic poles. Jian et al. utilised a pair of magnets to separate diamagnetic particles and cells in a ferrofluid flow [31]. The first magnet close to the channel focused the particle mixture into a single stream. The offset magnet deflected the $10-\mu \mathrm{m}$ from the 3- $\mu \mathrm{m}$ polystyrene particles as well as live yeast cells. However, operating the lab-on-chip (LOC) for a long time period is still challenging for negative magnetophoresis. Magnetic nanoparticles within the ferrofluid solution accumulate on the side of the channel due to positive magnetophoresis [32]. Visualisation of the cells becomes harder as the highly concentrated ferrofluid is opaque [4]. For a longer operation, the channel could be completely blocked, degrading its performance. By optimising the $\mathrm{pH}$ value, nanoparticles surfactants and tonicity, biocompatibility and colloidal stability can be improved [8].

Another solution for the accumulation of magnetic nanoparticles is introducing parallel ferrofluid streams with predefined concentrations. The low concentration towards the stronger magnetic field could prevent particle accumulation. Recently, Zhao et al. demonstrated on-chip sample preparation and separation of lowly concentrated cancer cells from white blood cells with a separation efficiency of $82 \%$ [33]. The authors introduced cell sample, ferrofluid and buffer solution through three different inlets. In the main channel, the cell sample instantly mixed with the ferrofluid and get separated to the buffer solution by the applied magnetic field gradient. The reduced exposure of cells to ferrofluid enhances the biocompatibility. Zhou et al. demonstrated two co-flowing fluids to focus and separate $2-\mu \mathrm{m}$ and $7-\mu \mathrm{m}$ of particles [34]. The $7-\mu \mathrm{m}$ particles in ferrofluid were focused on the fluid interfaces and maintained this path due to the co-flowing diamagnetic water streams. The smaller particles keep their initial trajectories and are separated from the mixture. The flow rate ratio of the two streams plays a vital role in the separation of these particles. In both cases, the parallel streams of ferrofluid and water have significantly different magnetic properties. 
In this paper, we utilise the benefits of negative magnetophoresis and consider the above challenges for the device design. We investigate the separation process of diamagnetic particles of 3.2- $\mu \mathrm{m}$ and $4.8-\mu \mathrm{m}$ of diameter in ferrofluid streams with different concentrations of magnetic nanoparticles. The streams with predefined concentrations promote negative magnetophoresis and reduce the migration of magnetic nanoparticles toward the magnet. Systematic investigation of the flow pattern, concentration of the solution and the magnetic field strength were carried out to optimise the separation performance.

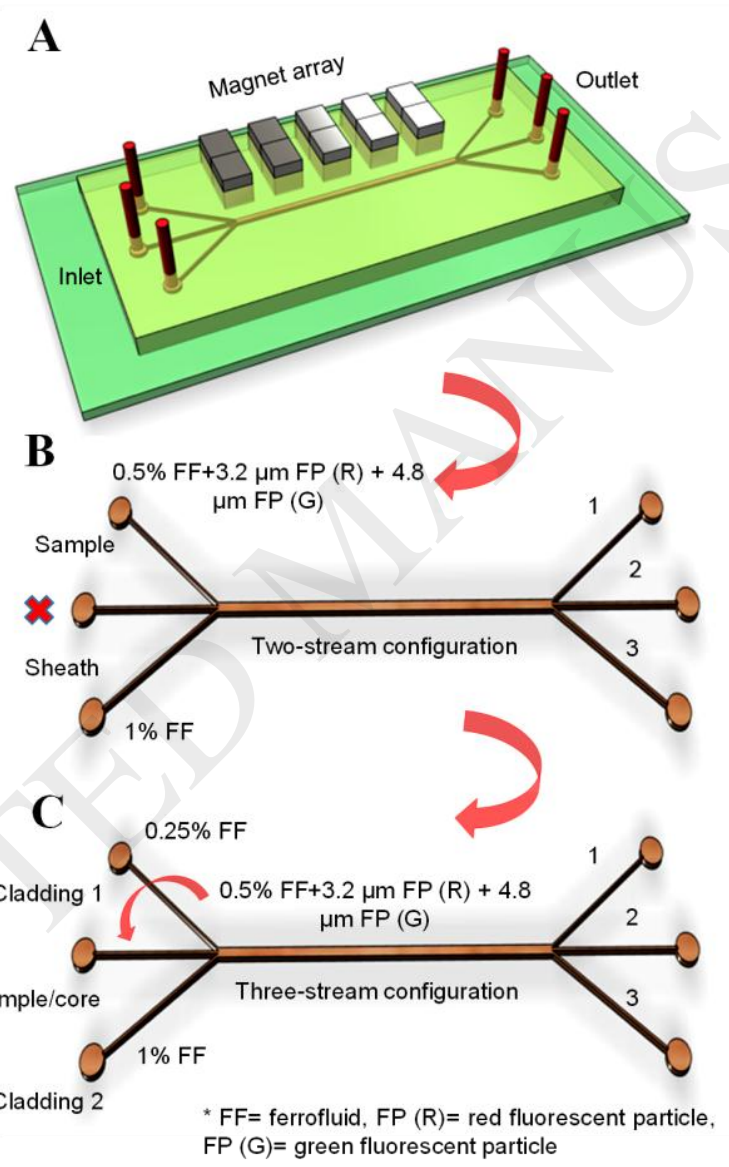

Fig. 1 Negative magnetophoretic separation of nonmagnetic particles with different sizes: $(A)$ Schematic diagram of the microfluidic device with the magnetic array; (B) Two-stream configuration and (C) Three-stream configuration.

\section{Material and methods}




\subsection{Basic design and working principle}

In the second part, $0.5 \%$ vol. ferrofluid in DI water mixed with the $3.2-\mu \mathrm{m}$ red, and 4.8$\mu \mathrm{m}$ green fluorescent polyethylene microspheres was introduced as the sample stream in a three-stream configuration, Fig. 1C. Moreover, $0.25 \%$ and $1.0 \%$ vol. ferrofluid without particles were used as cladding streams to focus the sample stream. Three precision syringe pumps (SPM-100, SIMTech Microfluidics Foundry) delivered the three streams to the device. The flow rate of the sample stream ranged between $0.5-2 \mu \mathrm{L} / \mathrm{min}$, while the cladding streams varied from $0.5-2 \mu \mathrm{L} / \mathrm{min}$. Because of the minute size difference between the two particles, we have to restrict the total flow to $6 \mu \mathrm{L} / \mathrm{min}$ to minimise the effect of convection and to enable efficient separation.

The diluted ferrofluid served as the paramagnetic medium. Diamagnetic particles of different sizes created magnetic holes with a mismatch in magnetic susceptibilities. The multistream ferrofluid flow reduces the accumulation of nanoparticles near the magnets, increasing the time span of the experiments [33]. The experiments were recorded over a period of 30 seconds to observe particle separation. An USB camera (Edmund Optics, Germany) was used to record the colour video at 10 frames per second through an inverted microscope (Nikon Eclipse TE-100). FITC (B-2A, EX 450-490), and TRITC (G-2A, Ex 510-560) filters were used to trace the green and red fluorescent particles through the $4 \times$-objective lens. The image was taken near the inlet to observe the focusing process of the particles. Particle separation was investigated at the outlets. Image J software (NIH, imagej.net) was used to convert the video into still images. Background noise was subtracted to evaluate the separation performance using a customised MATLAB (MathWorks) code. A fixed time period of 50 frames were evaluated to determine the separation efficiency. 

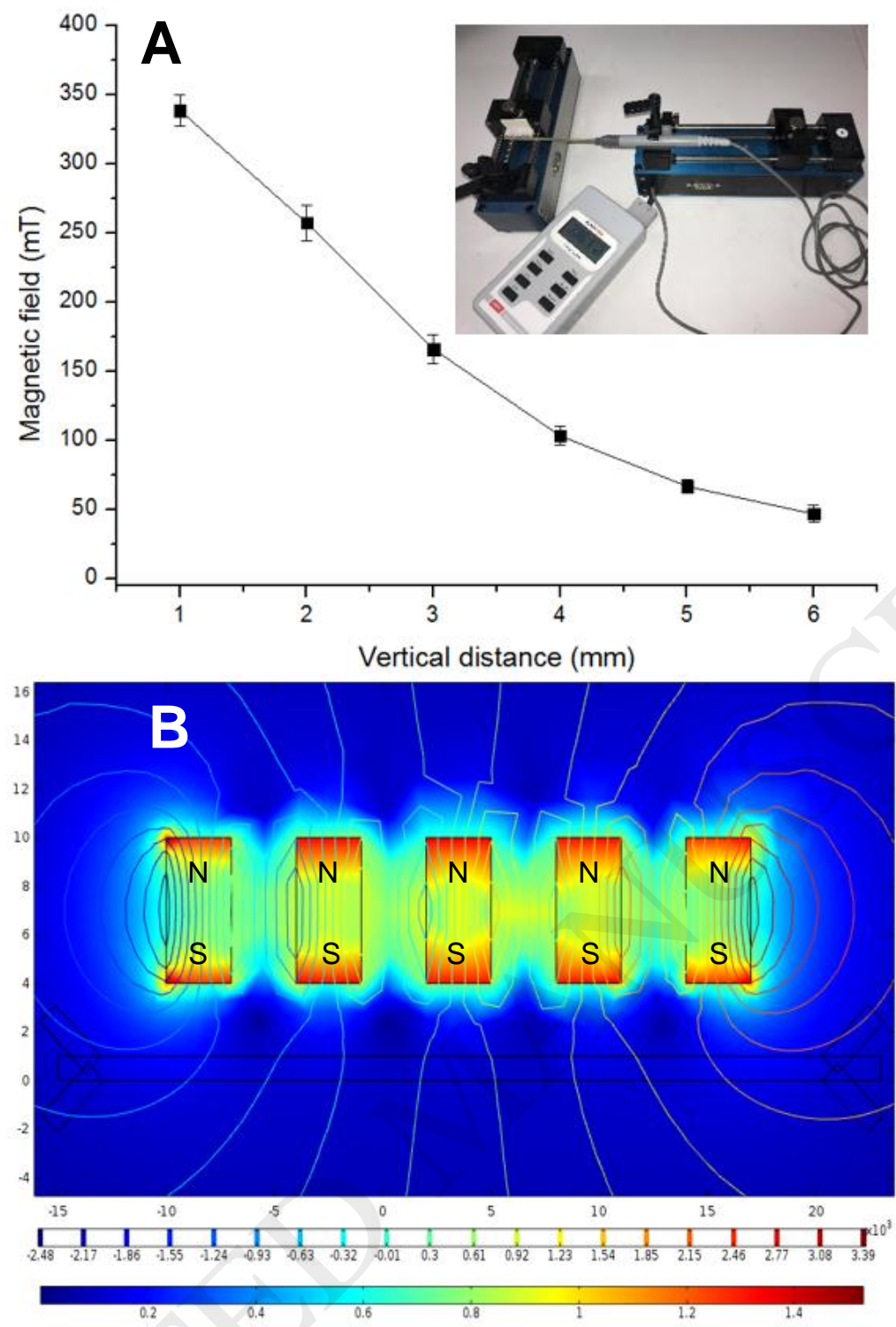

Fig. 3 (A) Magnetic flux density versus the distance of the fluidic channel, and (B) simulated magnetic flux density at a distance of $3 \mathrm{~mm}$ from the fluidic channel (repulsive force between magnets).

Five rectangular $\mathrm{NdFeB}$ magnets (grading N42, 3×6×3 mm, AFM Magnetics, Australia) were placed $3 \mathrm{~mm}$ apart from the separation channel to induce negative magnetophoresis. The magnet array was positioned in a frame made of PMMA, which was fabricated with a laser machining system (Trotec/Rayjet 300). Fig. 3A shows the flux density of the magnet versus the distance to the channel. The field strength of the magnet was calibrated by a Gauss meter (Hirst Magnetic Instruments Ltd., UK). COMSOL Multiphysics was used to simulate the flux distribution of the magnets, Fig. 3B. The repulsive force between each magnet 
(south-south or north-north) offers a quasi-uniform magnetic field. The magnetisation gradient generated by the ferrofluid concentration distribution is more controllable for the separation of particles with small size difference. Thus, the quasi-uniform field multiple permanent magnets can improve the separation performance in the channel for a wide range of flow rate ratio [37, 38]. Arrangement of attractive magnet pole (north-south or south-north) creates magnetic field gradient that pushes both the particles towards the magnetic field minima and trapping them in the channel. Thus an efficient separation is not achievable for a wide range of flow rate ratio in this magnet configuration. Supplementary Figure S1 presents the attractive flux distribution of the magnet array. Corresponding two-flow stream and three-flow stream particle separation at an optimum flow rate ratio are presented in

supplementary Figures S2, S3 respectively.

\subsection{Numerical simulations}

COMSOL Multiphysics 5.2 (COMSOL Inc., USA) was used to simulate the magnetic field distribution over the fluidic channel. Five $3 \times 6 \times 3 \mathrm{~mm}$ magnets were placed $3 \mathrm{~mm}$ apart from the main channel. The gap between each magnet was $3 \mathrm{~mm}$. A magnetic insulator was introduced around the system. The magnetisation was set as $1.6 \times 10^{6} \mathrm{Am}^{-1}$. Fig. $3 \mathrm{~B}$ shows the simulated magnetic flux distribution over the microchannel.

Particle trajectories were modelled by coupling the magnetic field, no current (MFNC), and creeping flow (SPF2) conditions with particle tracing for fluid flow (FPT). The model consists of three inlets, a long separation channel, and three outlets. The fluid is considered as incompressible. The wall has no-slip boundary condition with normal inflow velocity. The depth of the channel was considered by utilising the shallow channel approximation in the SPF2 model. The shallow fluidic channel had a thickness of $150 \mu \mathrm{m}$. Corresponding to the later experimental configurations, the simulation was conducted for two configurations. The 
two-stream configuration was considered for different flow rate ratios between the sheath and sample streams. The center inlet was blocked and switched off. The total flow rate ranged from 3 to $6 \mu \mathrm{L} / \mathrm{min}$. Next, the three-stream configuration with cladding stream 1 , core stream, and cladding stream 2 was simulated. The core stream has a flow rate ranging from 0.5 to $2.0 \mu \mathrm{L} / \mathrm{min}$. Both cladding streams were varied from 0.5 to $2.0 \mu \mathrm{L} / \mathrm{min}$ for each core flow rate. Two non-magnetic particles with a diameter of $3.2-\mu \mathrm{m}$ and $4.8-\mu \mathrm{m}$ were equally seeded in the core stream in both configurations. The particles have an identical density of $1050 \mathrm{~kg} / \mathrm{m}^{3}$. The particles were released at an interval of 0.2 seconds for a total time period of 20 seconds. A relative permeability of 1.51 was assigned to the ferrofluid of the model. The relative permeability of the particle was 0.2 .

Transport of diluted species (TDS) was coupled with the MFNC and SPF2 models to simulate the concentration distribution of the ferrofluid. The customized ferrofluid was introduced into the inlet with different concentrations. The diffusion coefficient was isotropic and fixed at the value of $1 \times 10^{-9} \mathrm{~m}^{2} / \mathrm{s}$. For the two-stream configuration, the sample and the sheath flow had a ferrofluid concentration of 0.05 and $0.1 \mathrm{~mol} / \mathrm{m}^{3}$, respectively representing the $0.5 \%$ and $1.0 \%$ vol. concentration in the experiments. The centre inlet was closed. For the three-stream configuration, cladding 1, sample/core, and cladding 2 have a ferrofluid concentration of $0.025,0.05$, and $0.1 \mathrm{~mol} / \mathrm{m}^{3}$ representing $0.25 \%, 0.5 \%$ and $1.0 \%$ vol. concentration in the experiments, respectively. 

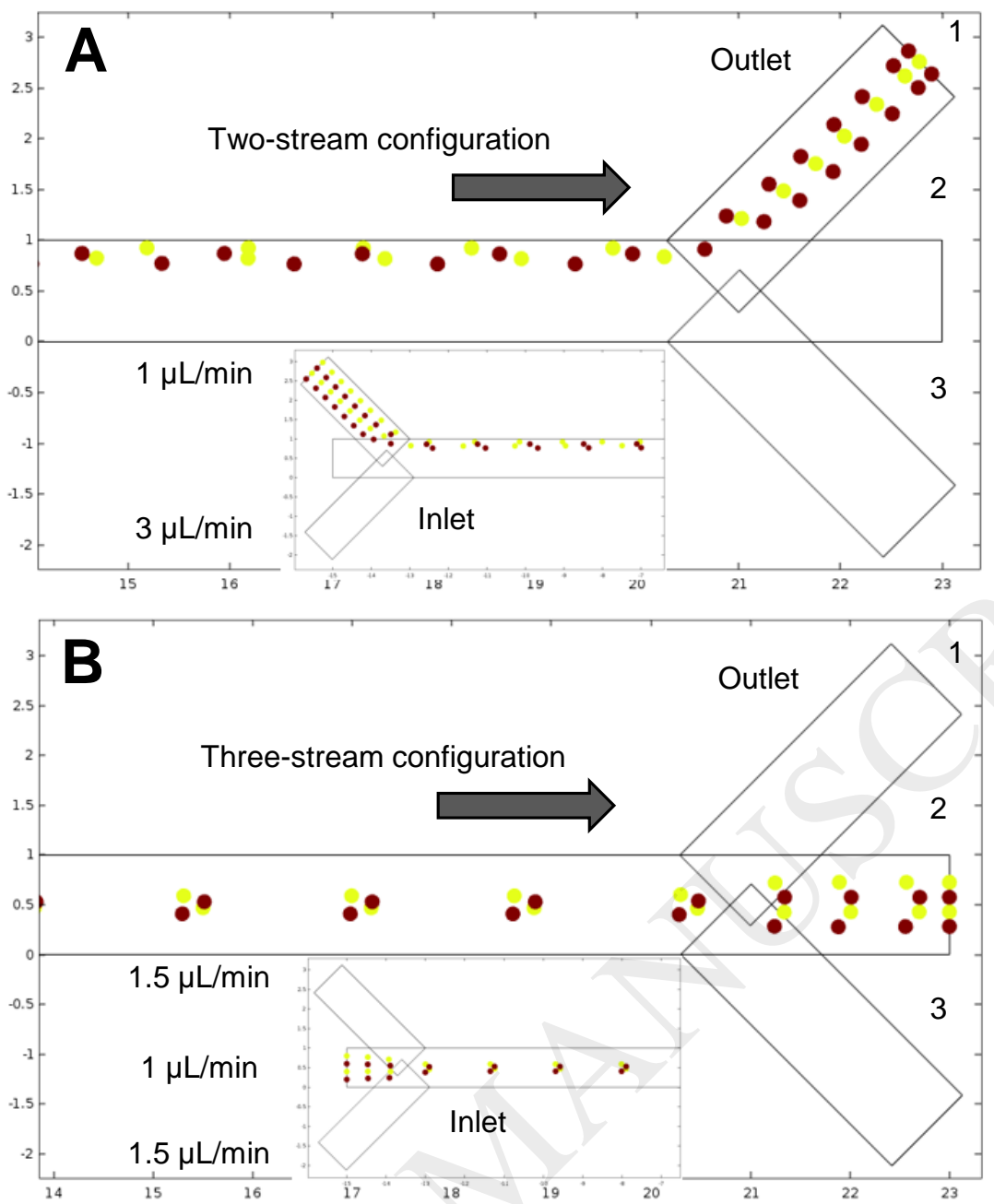

Fig. 4 Numerical simulation of two particle sizes without magnetic field for $(A)$ two-stream configuration and (B) three-stream configuration.

Fig. 8 shows the corresponding normalised magnetophoretic force (Eqn. 8, supplementary S1) for the 3.2- $\mu \mathrm{m}$, and 4.8- $\mu \mathrm{m}$ particles across the channel width. The 3.2- $\mu \mathrm{m}$ diamagnetic particle exhibits a repulsive force between $0.95-2.44 \mathrm{pN}$ across the channel width, Fig. 8A. The force gradually increases toward the magnet arrays. Interestingly, the magnetophoretic force at magnet 1 , and 5 shows higher magnitude compared to the magnet at 2-4 locations. As magnet 1 , and 5 are at both ends of the array, the flux line distribution exhibits repulsive force from its 
adjacent neighbours as well as an attractive force towards its own opposite poles (Fig. 3B). As a consequence, the magnetic field strength is higher at magnet 1 and 5 across the channel width. This higher force initiates focusing and separation of the particles within the channel. The 4.8$\mu \mathrm{m}$ particle shows a higher magnetophoretic force of 3.3-8 $\mathrm{pN}$ throughout the channel, Fig. 8B. The active drag force for both of the particle shows identical magnitude across the channel width (Eqn. 6, supplementary S1). The normalized drag force for the 3.2- $\mu \mathrm{m}$, and $4.8-\mu \mathrm{m}$ particles are between $0.95-2.44 \mathrm{pN}$, and $3.3-8 \mathrm{pN}$ respectively that agrees with the force balance conditions (supplementary Figure S4).

\section{Results and discussion}

Figure 10 presents the experimental particle separation for the two-stream and three-stream configurations. The separation of the two particle types was possible. Furthermore, the multistream ferrofluid flow promoted this separation as predicted by the simulation. The simulated optimal flow rate ratio was confirmed by the experiment. A lower flow rate of the sample/core stream focuses the sample at the channel inlet. A slight change in focus caused by air bubbles can drastically affect the separation performance [22]. Before the experiment, the devices were rinsed with DI water, dried in an oven at $80^{\circ} \mathrm{C}$ for 1 hour, and subsequently exposed to UV to minimise air bubbles during the experiments. The syringe pump was connected to the device by two identical Teflon tubes to avoid any hydrostatic pressure difference. Particle separation was normalized by analysing each frame of the video with ImageJ software.

For the flow rate ratio of $1: 4,60 \%$ of the $3.2-\mu \mathrm{m}$ particles pass through outlet 3 . The separation efficiency of $4.8-\mu \mathrm{m}$ particles is $59 \%$ at outlet 2 . For an increased flow rate ratio of 1:5, the efficiency become 58\%, and 58\% respectively. Probably for a higher total flow rate of more than $4 \mu \mathrm{L} / \mathrm{min}$, the drag force dominates over the magnetophoretic force. The particles 
follow the hydrodynamic trajectory and reduce the separation performance. The experimental data show similar separation pattern as predicted by the simulation in Fig. 9A.

Fig. 10B shows the separation performance of the three-stream configuration for different flow rate ratios. For the flow rate ratio between cladding 1, core and cladding 2 of 1.5:0.5:1.5, the maximum separation efficiency were $75 \%$, and $56 \%$ for $3.2-\mu \mathrm{m}$ and $4.8-\mu \mathrm{m}$ particles, respectively. The separation performance increases to $78 \%$ and $75 \%$ for the 3.2 and 4.8- $\mu \mathrm{m}$ particles respectively at a flow ratio of 1.5:1:1.5 $\mu \mathrm{L} / \mathrm{min}$. Further increasing the flow ratio to $2: 1.5: 2 \mu \mathrm{L} / \mathrm{min}$ reduces the separation performance to $73 \%$ and $77 \%$, respectively. The separation results are $76 \%$, and $41 \%$ for the flow rate ratio of 2:2:2. At a total flow rate above $5 \mu \mathrm{L} / \mathrm{min}$, the drag force dominates the trajectories of the particles.

Most of the 3.2- $\mu \mathrm{m}$ particles pass through outlet 1 . This is a deviation from the simulated results shown in Fig. 9B. The 3.2- $\mu \mathrm{m}$ particles behaved in the experiment as magnetic particles that move towards the magnetic field maxima. The results for this behaviour could be the attachment of magnetic nanoparticles on the particles. Possibly, the amount of magnetic nanoparticles attached to the smaller $3.2-\mu \mathrm{m}$ particles are enough to pull them towards the magnetic field maxima [39]. Furthermore, the simulated data indicate a better performance compared to experimental data.

\section{Conclusion}

Negative magnetophoresis is a promising technique for the separation of diamagnetic particles of different sizes. The concept reported here is a convenient mean for the separation of two or more particle types with a small size difference. We observed the effect of multi-stream ferrofluid flow to separate diamagnetic particles of different sizes. Numerical simulation was conducted to study the distribution of the magnetic field, the effect of the flow rates, and the concentration distribution of ferrofluid in a multi-stream configuration. In our experiments, the 
optimum total flow rate for both of two-stream and three-stream configurations was approximately $4 \mu \mathrm{L} / \mathrm{min}$. The ferrofluid concentration ranges from $0.25 \%, 0.5 \%$, and $1 \%$ vol. ratio. The designated particle mixture flowed through a relatively higher concentration of ferrofluid to promote the negative magnetophoresis. Sheath/cladding flow was used to focus the particle mixture into a single stream. The experimental data show an excellent separation performance of diamagnetic particles by the three-stream configuration. In the two-stream configuration, the normalized separation efficiency was $68 \%$, and $72 \%$ for the $3.2-\mu \mathrm{m}$ red and 4.8- $\mu \mathrm{m}$ green particles respectively at a flow rate ratio of $1: 3$. In the three-stream configuration, the maximum separation efficiency was $78 \%$ and $75 \%$, respectively at a flow rate ratio of 1.5:1:1.5. The $3.2-\mu \mathrm{m}$ particles flow through outlet 1 towards to the magnet array. This behaviour may be caused by the attachment of the magnetic nanoparticles of the ferrofluid on the microparticles. With further improvement and the use of biocompatible ferrofluids, the developed methods can be utilized to sort biological cells and bacteria with small size difference. The present study serves as a guide for the separation of particles and cells with a diameter of less than $5 \mu \mathrm{m}$. In the future, the design and the dimension will be tuned to improve the separation performance for a wider range of size.

\section{Acknowledgments}

\section{Author biographies}

Munaz Ahmed is a PhD student at Queensland Micro- and Naotechnology Centre at Griffith University.

Muhammad J. A. Shiddiky is a senior lecturer and National Health and Medical Research Council (NHMRC) CDF Fellow in the School of Natural Sciences at Griffith University. He obtained his Ph.D. from Pusan national University, South Korea in 2007. Following his Ph.D. work, he was a Postdoctoral Fellow at Monash University and Australia Research Council (ARC) DECRA Fellow at the University of Queensland. His research focuses on the development of new technologies and devices for clinical diagnostics. Currently he is involved in developing functional nanomaterial based portable devices for diagnosing, measuring, and treating cancer, infectious, and neurodegenerative diseases.

Nam-Trung Nguyen is a professor and the director of Queensland Micro- and Nanotechnology Centre, Griffith University, Australia. He received his Dipl-Ing, Dr-Ing and Dr-Ing habil degrees from the Chemnitz University of Technology, Germany, in 1993, 1997 and 2004, respectively. He was a 
postdoctoral research engineer at the Berkeley Sensor and Actuator Center, University of California, Berkeley, USA. From 1999 to 2013, he was a research fellow, assistant professor and associate professor at Nanyang Technological University, Singapore. Dr. Nguyen has published over 330 journal papers and several books on micro- fluidics and nanofluidics.

The authors acknowledge funding support from Australian Research Council through the grant DP180100055. 


\section{Reference}

[1] A. Munaz, M.J. Shiddiky, N.-T. Nguyen, Recent advancements and current challenges in magnetophoresis-based micro magnetofluidics, Biomicrofluidics, 12(2018) 031501-23.

[2] M. Hejazian, W. Li, N.-T. Nguyen, Lab on a chip for continuous-flow magnetic cell separation, Lab on a Chip, 15(2015) 959-70.

[3] G. Zhu, N. Trung Nguyen, Particle sorting in microfluidic systems, Micro and Nanosystems, 2(2010) 202-16.

[4] W. Zhao, R. Cheng, J.R. Miller, L. Mao, Label-Free Microfluidic Manipulation of Particles and Cells in Magnetic Liquids, Advanced Functional Materials, 26(2016) 3916-32.

[5] R.E. Rosensweig, Ferrohydrodynamics: Courier Corporation; 2013.

[6] N. Pamme, Magnetism and microfluidics, Lab on a Chip, 6(2006) 24-38.

[7] W. Zhao, T. Zhu, R. Cheng, Y. Liu, J. He, H. Qiu, et al., Label-Free and Continuous-Flow Ferrohydrodynamic Separation of HeLa Cells and Blood Cells in Biocompatible Ferrofluids, Advanced functional materials, 26(2016) 3990-8.

[8] W. Zhao, R. Cheng, B.D. Jenkins, T. Zhu, N.E. Okonkwo, C.E. Jones, et al., Label-free ferrohydrodynamic cell separation of circulating tumor cells, Lab on a Chip, 17(2017) 3097-111.

[9] R. Cheng, T. Zhu, L. Mao, Three-dimensional and analytical modeling of microfluidic particle transport in magnetic fluids, Microfluidics and nanofluidics, 16(2014) 1143-54.

[10] P.-H. Shih, J.-Y. Shiu, P.-C. Lin, C.-C. Lin, T. Veres, P. Chen, On chip sorting of bacterial cells using sugar-encapsulated magnetic nanoparticles, Journal of Applied Physics, 103(2008) 07A316.

[11] N. Pamme, C. Wilhelm, Continuous sorting of magnetic cells via on-chip free-flow magnetophoresis, Lab on a Chip, 6(2006) 974-80.

[12] A. Munaz, H. Kamble, M.J. Shiddiky, N.-T. Nguyen, Magnetofluidic micromixer based on a complex rotating magnetic field, RSC Advances, 7(2017) 52465-74.

[13] N. Xia, T.P. Hunt, B.T. Mayers, E. Alsberg, G.M. Whitesides, R.M. Westervelt, et al., Combined microfluidic-micromagnetic separation of living cells in continuous flow, Biomedical microdevices, 8(2006) 299.

[14] J.J. Wilbanks, G. Kiessling, J. Zeng, C. Zhang, T.-R. Tzeng, X. Xuan, Exploiting magnetic asymmetry to concentrate diamagnetic particles in ferrofluid microflows, Journal of Applied Physics, 115(2014) 044907.

[15] S.B.N. Gourikutty, C.-P. Chang, P.D. Puiu, Microfluidic immunomagnetic cell separation from whole blood, Journal of Chromatography B, 1011(2016) 77-88.

[16] L. Liang, X. Xuan, Continuous sheath-free magnetic separation of particles in a U-shaped microchannel, Biomicrofluidics, 6(2012) 044106.

[17] S. Yan, J. Zhang, D. Yuan, Q. Zhao, J. Ma, W. Li, High-throughput, sheathless, magnetophoretic separation of magnetic and non-magnetic particles with a groove-based channel, Applied Physics Letters, 109(2016) 214101.

[18] T.T. Puck, P.I. Marcus, S.J. Cieciura, Clonal growth of mammalian cells in vitro: growth characteristics of colonies from single HeLa cells with and without a" feeder" layer, Journal of Experimental Medicine, 103(1956) 273-84.

[19] K. de Jong, R.K. Emerson, J. Butler, J. Bastacky, N. Mohandas, F.A. Kuypers, Short survival of phosphatidylserine-exposing red blood cells in murine sickle cell anemia, Blood, 98(2001) 1577-84.

[20] V. Murlidhar, L. Rivera-Báez, S. Nagrath, Affinity Versus Label-Free Isolation of Circulating Tumor Cells: Who Wins?, small, 12(2016) 4450-63.

[21] Y. Chen, P. Li, P.-H. Huang, Y. Xie, J.D. Mai, L. Wang, et al., Rare cell isolation and analysis in microfluidics, Lab on a Chip, 14(2014) 626-45.

[22] M. Antfolk, T. Laurell, Continuous flow microfluidic separation and processing of rare cells and bioparticles found in blood-A review, Analytica chimica acta, 965(2017) 9-35. 
[23] N.M. Anstey, B. Russell, T.W. Yeo, R.N. Price, The pathophysiology of vivax malaria, Trends in parasitology, 25(2009) 220-7.

[24] R.K. Vadivelu, H. Kamble, A. Munaz, N.-T. Nguyen, Liquid marbles as bioreactors for the study of three-dimensional cell interactions, Biomedical microdevices, 19(2017) 31.

[25] L. Liang, C. Zhang, X. Xuan, Enhanced separation of magnetic and diamagnetic particles in a dilute ferrofluid, Applied Physics Letters, 102(2013) 234101.

[26] T. Zhu, F. Marrero, L. Mao, Continuous separation of non-magnetic particles inside ferrofluids, Microfluidics and nanofluidics, 9(2010) 1003-9.

[27] P. Yeh, Z. Dai, X. Yang, M. Bergeron, Z. Zhang, M. Lin, et al., An efficient spiral microchannel for continuous small particle separations, Sensors and Actuators B: Chemical, 252(2017) 606-15.

[28] I. Johnston, M. McDonnell, C. Tan, D. McCluskey, M. Davies, M. Tracey, Dean flow focusing and separation of small microspheres within a narrow size range, Microfluidics and Nanofluidics, 17(2014) 509-18.

[29] T. Zhu, R. Cheng, Y. Liu, J. He, L. Mao, Combining positive and negative magnetophoreses to separate particles of different magnetic properties, Microfluidics and nanofluidics, 17(2014) 973-82.

[30] M. Hejazian, N.-T. Nguyen, Magnetofluidic concentration and separation of non-magnetic particles using two magnet arrays, Biomicrofluidics, 10(2016) 044103.

[31] J. Zeng, Y. Deng, P. Vedantam, T.-R. Tzeng, X. Xuan, Magnetic separation of particles and cells in ferrofluid flow through a straight microchannel using two offset magnets, Journal of Magnetism and Magnetic Materials, 346(2013) 118-23.

[32] A. Skjeltorp, One-and two-dimensional crystallization of magnetic holes, Physical Review Letters, 51(1983) 2306.

[33] W. Zhao, R. Cheng, S.H. Lim, J.R. Miller, W. Zhang, W. Tang, et al., Biocompatible and Label-Free Separation of Cancer Cells of Cell Culture Lines from White Blood Cells in Ferrofluids, Lab on a Chip, (2017).

[34] R. Zhou, C. Wang, Multiphase ferrofluid flows for micro-particle focusing and separation, Biomicrofluidics, 10(2016) 034101.

[35] A. Munaz, R.K. Vadivelu, J.S. John, M. Barton, H. Kamble, N.-T. Nguyen, Three-dimensional printing of biological matters, Journal of Science: Advanced Materials and Devices, 1(2016) 1-17.

[36] A. Munaz, R.K. Vadivelu, J.A. St John, N.-T. Nguyen, A lab-on-a-chip device for investigating the fusion process of olfactory ensheathing cell spheroids, Lab on a Chip, 16(2016) 2946-54.

[37] A. Munaz, B.-C. Lee, G.-S. Chung, A study of an electromagnetic energy harvester using multi-pole magnet, Sensors and Actuators A: Physical, 201(2013) 134-40.

[38] A. Munaz, G.-S. Chung, An electromagnetic energy harvester based on multiple magnet scavenging power from low frequency vibration, Microsystem Technologies, (2015) 1-9.

[39] M. Benelmekki, L.M. Martinez, J. Andreu, J. Camacho, J. Faraudo, Magnetophoresis of colloidal particles in a dispersion of superparamagnetic nanoparticles: theory and experiments, Soft Matter, 8(2012) 6039-47.

Figure 1 shows the schematic of the microfluidic device and the two configurations under investigation. Figure 2 shows the corresponding SEM images of the channel surface at different locations of the microfluidic chip. The device consists of three inlets, a main channel to initiate the separation, and three outlets. A three-dimensional (3D) model of the device was designed with 123D (Autodesk, USA) software. The model was then converted into Standard 
Tessellation Language (STL) format. We then used a commercial 3D printer (Nobel 1.0A, XYZ printing, USA) to fabricate the master mold from the STL file [35]. The main microchannel has a length of $L=30 \mathrm{~mm}$, a width of $W=1 \mathrm{~mm}$, and a thickness of approximately $D=150 \pm 10 \mu \mathrm{m}$. We used ethyl alcohol to remove any residual resins from the moulds. The mould was subsequently cured in ultraviolet (UV) light (Tray-Lux M5, Taiwan) and further rinsed with DI water. Polydimethylsiloxane (PDMS) prepolymer was mixed with the crosslinker at 10:1 ratio. The PDMS was degassed and poured into the master mould [36]. The PDMS was degassed for another 30 min to remove air bubbles. The mould was then placed in an oven at $80^{\circ} \mathrm{C}$ for 5 hours for curing. The replicated PDMS was taken off from the mould, and inlet/outlet access holes were punched. The replicated PDMS was further cleaned to remove any residual dirt. The PDMS was then bonded to a clean glass slide after being treated for 45 seconds in an air plasma.

The experiment was carried out in two parts. In the first part, particles of two different sizes were separated in a two-stream configuration, Fig. 1B. A water-based ferrofluid (EMG 707 , Ferrotech, USA) was diluted to $0.5 \%$ volume concentration $(\varphi=0.5 \%$ vol. $)$ with DI water. We then mixed 3.2- $\mu \mathrm{m}$ red and $4.8-\mu \mathrm{m}$ green fluorescent polyethylene microbeads $(1.00 \mathrm{~g} / \mathrm{cc}$, Thermoscientific Inc.) with this ferrofluid solution to serve as the sample, which is then delivered by a syringe pump into one of the two inlets, Fig. 1B. Diluted ferrofluid ( $\varphi=1 \%$ vol.) without florescent particle was used as the sheath stream to focus the sample stream. The centre inlet was closed in this configuration. Two precision syringe pumps (SPM-100, SIMTech Microfluidics Foundry) delivered the two streams. Different flow rate ratios for the sample and sheath streams were tested for the range of 3 to $6 \mu \mathrm{L} / \mathrm{min}$. 


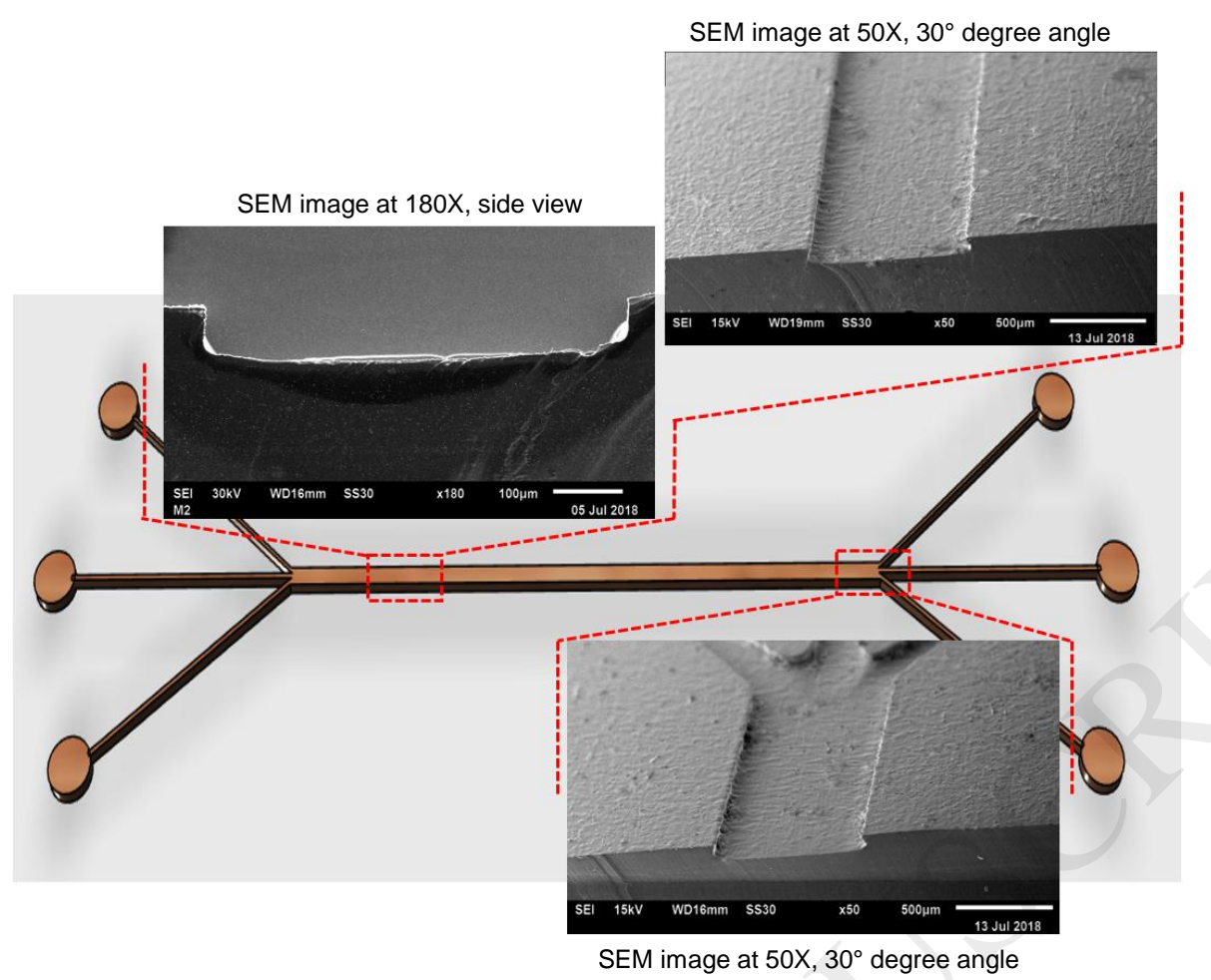

Fig. 2 SEM images of the channel surface at different location of the microfluidic chip. 


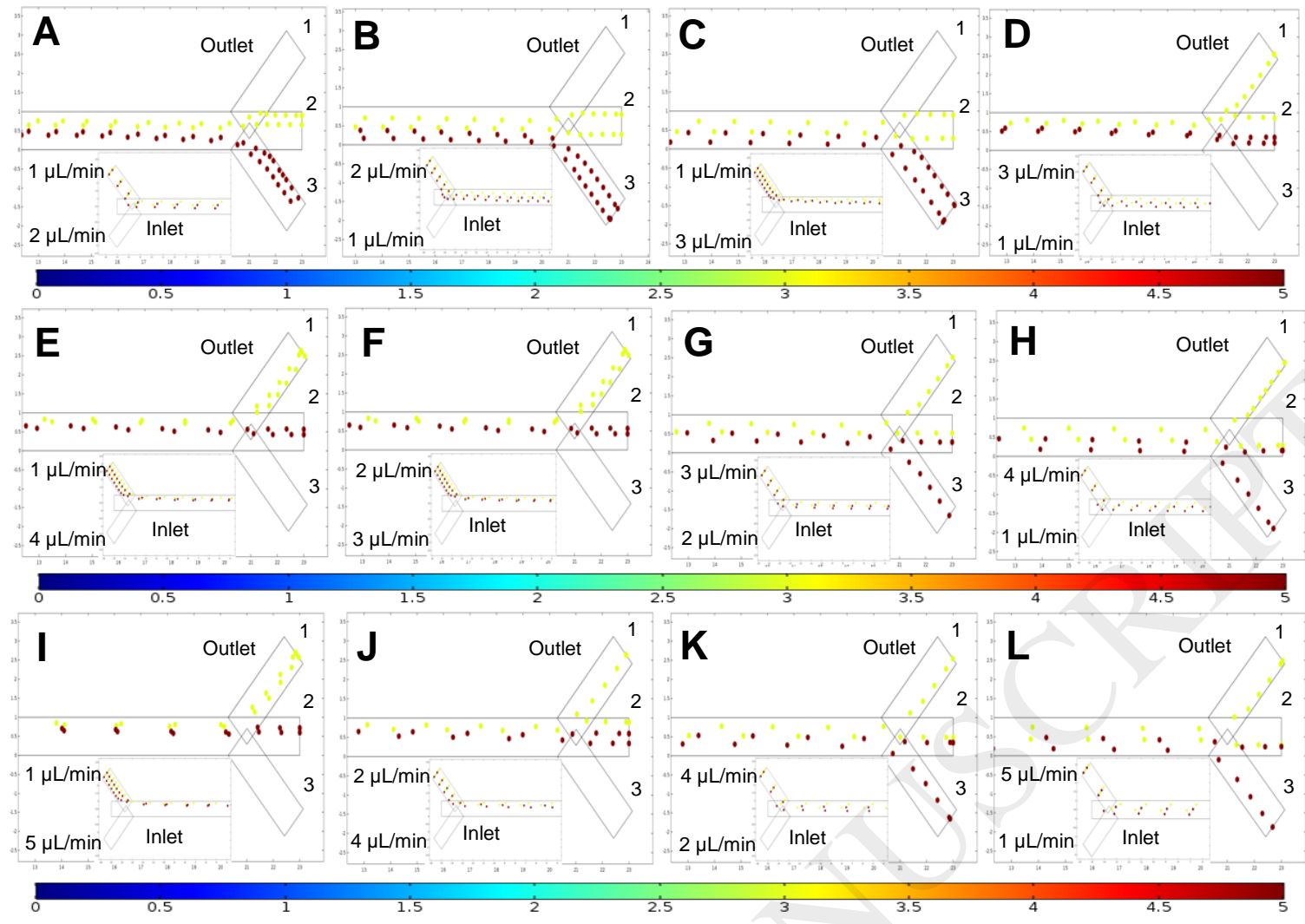

Fig. 5 Numerical simulation of the separation of two diamagnetic particle types by negative magnetophoresis for a total flow of (A-B) $3 \mu \mathrm{L} / \mathrm{min}$, (C-D) $4 \mu \mathrm{L} / \mathrm{min}$, (E-H) $5 \mu \mathrm{L} / \mathrm{min}$, and (I-L) $6 \mu \mathrm{L} / \mathrm{min}$. Flow rate ratio for sample and sheath flows are presented for each condition. The colour bar presents the diameter of the particle $(\mu \mathrm{m})$.

Fig. 4 shows the trajectories for the 3.2- $\mu \mathrm{m}$ and $4.8-\mu \mathrm{m}$ particles under an optimal flow rate condition. If the magnetic field is not present in the system, the particles do not experience any magnetophoretic force. Both particle follow their initial trajectories by hydrodynamic drag force. For the two-stream configuration, the particle mixture passes through outlet 1 . For the three-stream configuration, the particles follow the centre stream and exit through outlet 2 . In an external magnetic field, the particle defections are initiated by the magnetophoretic force. The deflection of the particle depends on its size, flow rate ratio and the concentration of the surrounding medium. The larger particle experiences higher magnetophoretic and drag force. Both diamagnetic particles move toward the magnetic field minima as the result of negative 
magnetophoresis. Moreover, the size variation among the particle creates different magnetophoretic forces that initiate the separation. The separation efficiency can be further

tuned by optimizing the flow rate ratio.

Fig. 5 shows the numerical trajectories of the two diamagnetic particles. For the twostream flow, the sample and sheath streams were varied at different flow ratio keeping the total flow rate between 3 and $6 \mu \mathrm{L} / \mathrm{min}$. Fig. 5 A-B shows the particle trajectories for a total flow rate of $3 \mu \mathrm{L} / \mathrm{min}$. Both flow rate ratios of 1:2 and 2:1 result in an effective separation of 3.2$\mu \mathrm{m}$ particles from the $4.8-\mu \mathrm{m}$ particle. However, the $3.2-\mu \mathrm{m}$ particle passes the edge of outlet 2 in Fig. 5A due to the dominant sheath stream. For the flow rate ratio of 1:3 (Fig. 5C), effective particle separation is visible. However, at the flow rate ratio of 3:1 (Fig. 5D), particle focusing at the inlet becomes unstable. The mixture solution in the sample stream occupies more space in the channel. The smaller particles tend to pass through both outlets 1 and 2 , reducing the separation performance. Figures $5 \mathrm{E}-\mathrm{H}$ show the trajectory of the particle for a total flow rate of $5 \mu \mathrm{L} / \mathrm{min}$. The particle is well focused at the inlet position until the sample stream is smaller than the sheath stream. The magnetophoretic force actively influences the well-focused particle of different sizes. Particle separation is visible in Figs. 5E and 5F. When the sample stream is wider than the sheath stream (Figs. $5 \mathrm{G}$ and $5 \mathrm{H}$ ), the particle mixture occupies more area at the inlet leading to a reduction of separation efficiency. Similarly, for a sample to sheath stream ratio of 1:5 and 2:4, the particles are focused at the inlet, Figs. 5I and 5J. Effective separation between the two diamagnetic particles are visible. If the sample stream is wider than the sheath stream (Figs. 5K and 5L) magnetophoretic force cannot effectively influence the unfocused particle. Particle separation efficiency decreases. Particles of different sizes can be separated effectively with a focused and narrow sample stream. 
Fig. 6 shows the numerical trajectory of the particles for the three-stream configuration. The sample/core stream varied between 0.5 and $2 \mu \mathrm{L} / \mathrm{min}$. Figs. 6 A-D show a fixed core stream flow rate of $0.5 \mu \mathrm{L} / \mathrm{min}$ sandwiched between two cladding streams of $0.5-2 \mu \mathrm{L} / \mathrm{min}$. The results show a gradual deflection of 3.2 and $4.8-\mu \mathrm{m}$ of particles from the core stream. However, the magnetophoretic force acting on the $4.8-\mu \mathrm{m}$ particle is clearly dominant. The core of $0.5 \mu \mathrm{L} / \mathrm{min}$ and the cladding of 0.5 to $1 \mu \mathrm{L} / \mathrm{min}$ are insufficient to drag the particle out of the channel, Fig. 6A, B. However, the 3.2- $\mu$ m particles follow the magnetic field minima and pass through outlet 3. The higher cladding flow rates of 1.5 and $2 \mu \mathrm{L} / \mathrm{min}$ (Fig. 6C, D) focuses the particles at the inlet and allows for successful separation.

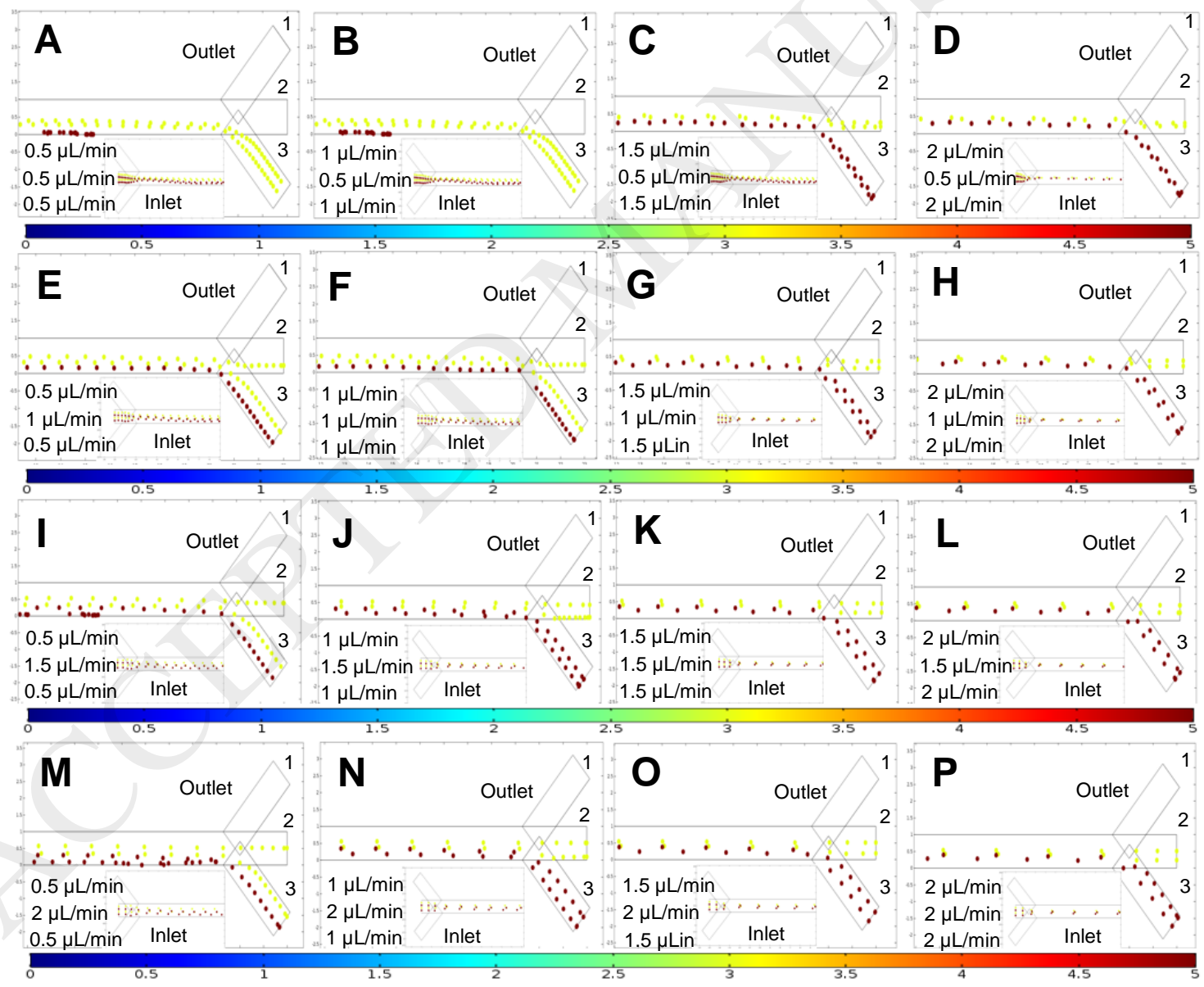

Fig. 6 Numerical simulation of the separation of two diamagnetic particle types separated by negative magnetophoresis for a sample/core flow of (A-D) $0.5 \mu \mathrm{L} / \mathrm{min},(\mathrm{E}-\mathrm{H}) 1 \mu \mathrm{L} / \mathrm{min},(\mathrm{I}-\mathrm{L}) 1.5 \mu \mathrm{L} / \mathrm{min}$, and (MP) $2 \mu \mathrm{L} / \mathrm{min}$. The flow rate ratio for sample/core and cladding flows are presented for each condition. The colour bar presents the diameter of the particle $(\mu \mathrm{m})$. 

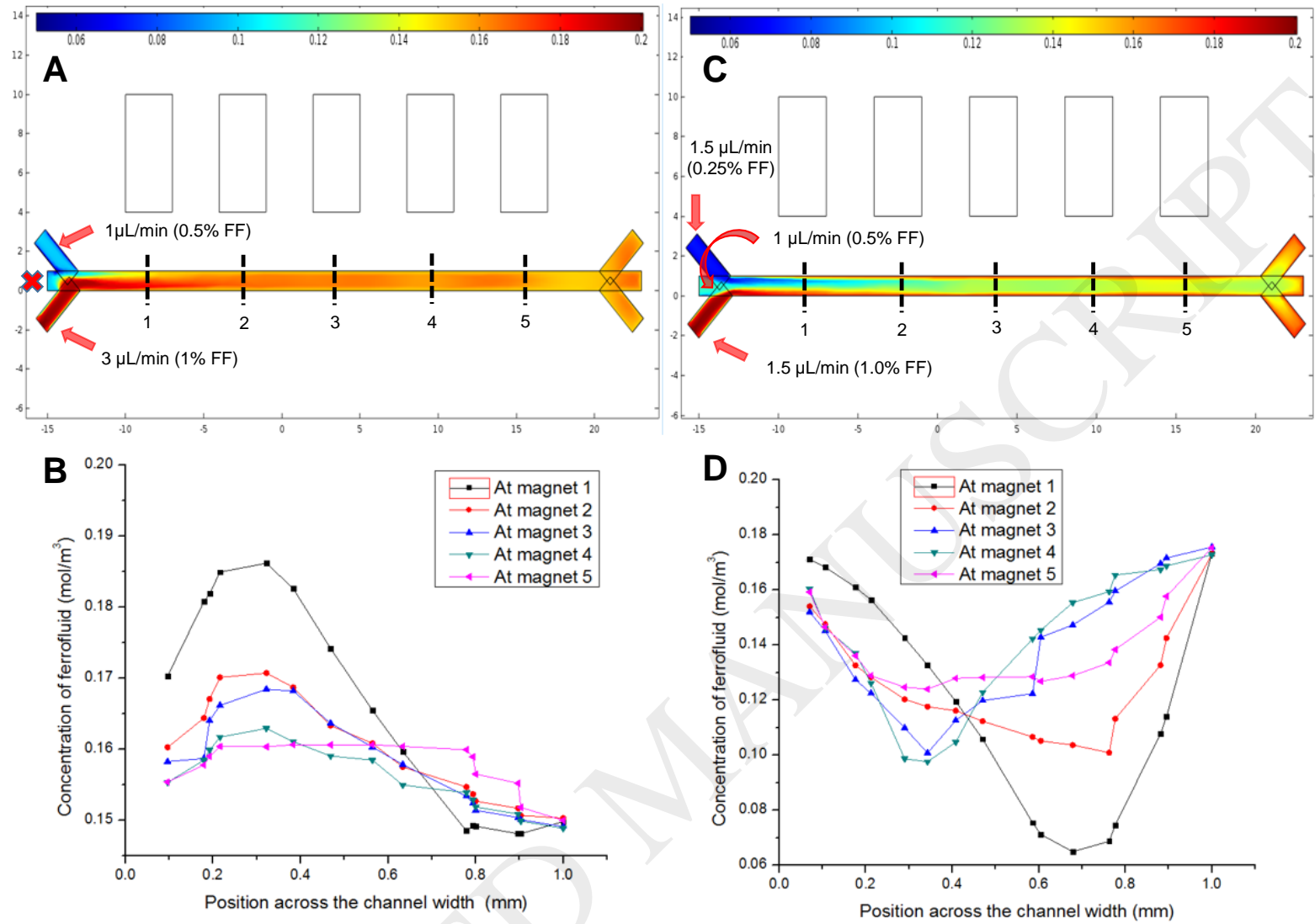

Fig. 7 Numerical results of concentration distribution for (A-B) two-stream configuration, and their position across the channel width, and (C-D) three-stream configuration and their position across the channel width.

As the core stream increases to $1 \mu \mathrm{L} / \mathrm{min}$ (Fig. $6 \mathrm{E}-\mathrm{H}$ ), particle clogging is not observed for the $4.8-\mu \mathrm{m}$ particles. If the cladding is smaller or equal to the core stream, both $3.2-\mu \mathrm{m}$ and 4.8- $\mu \mathrm{m}$ particles are seen at outlet 3 , Fig. $6 \mathrm{E}, \mathrm{F}$. Further increasing the cladding stream to 1.5 and $2 \mu \mathrm{L} / \mathrm{min}$ improves focusing of the particle sample. An effective separation of the particle is visible in Fig. 6G, H. Fig. 6I shows clogging of some 4.8- $\mu$ m particle for the core stream of $1.5 \mu \mathrm{L} / \mathrm{min}$. The 3.2- $\mu \mathrm{m}$ particles are visible at outlet 2, Fig. $6 \mathrm{~J}$. Efficient separation happens if the core flow rate is equal to or higher than that of the cladding stream, Fig. 6K, L. Figures $6 \mathrm{M}-\mathrm{P}$ show results of the core stream of $2 \mu \mathrm{L} / \mathrm{min}$ sandwiched between two cladding with a 
flow rate ranging from 0.5 to $2 \mu \mathrm{L} / \mathrm{min}$. Wall attachment is observed for $4.8-\mu \mathrm{m}$ particles (Fig. $6 \mathrm{M})$ as they deflect towards a relatively narrow cladding stream. Similarly, wall attachment is observed for 3.2- $\mu \mathrm{m}$ particles in Fig $6 \mathrm{~N}$. The separation of the particles is more efficient if they are more focused in the inlet region, Figs. $60, \mathrm{P}$. The simulation suggests that for an efficient

separation of particles, particle focusing, and the flow rate ratio are the key factors.

Fig. 7 shows the concentration distribution of the ferrofluid coupled with the magnetic field for the two-stream and three-stream configurations. The magnets create a strong nonuniform magnetic field around the separation channel. In the two-stream configuration, the ferrofluid streams mixes and reduces the concentration gradient, Fig. 7A. The concentration distribution across the width of the channel at 5 different positions corresponds to each of the magnets are shown in Fig. 7B. The concentration gradient becomes homogeneous close to the outlets. Because of the homogeneity of the ferrofluid, we expect that particle separation by negative magnetophoresis to be less efficient in the actual experiments.
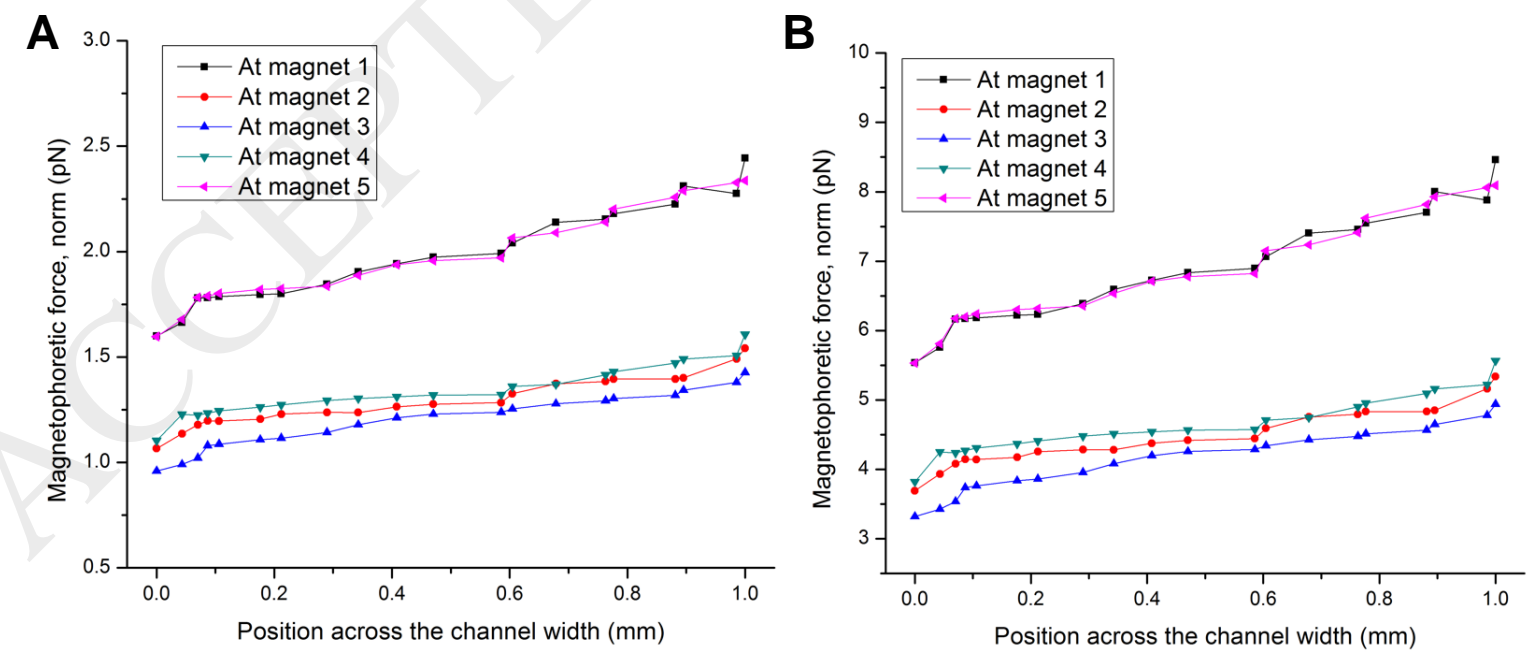

Fig. 8 Normalised magnetophoretic force across the channel width for (A) 3.2- $\mu$ m particles, and (B) $4.8-\mu \mathrm{m}$ particles. 
Fig. 7C shows the concentration distribution of ferrofluid for the three-stream configurations. High concentration is observed at cladding 2 near the inlet. This high concentration maintains on the side wall of the microchannel until the outlet. Furthermore, the accumulation of high ferrofluid concentration is observed close to the region with a strong magnetic field. Fig. 7D depicts the concentration distribution across the width for 5 different locations over the microchannel. The higher concentrations are clearly observed at the cladding throughout the channel. This concentration gradient promotes negative magnetophoresis over the channel length. Thus, under real experimental condition, particle separation with threestream configuration is expected to be more efficient than the two-stream configuration.
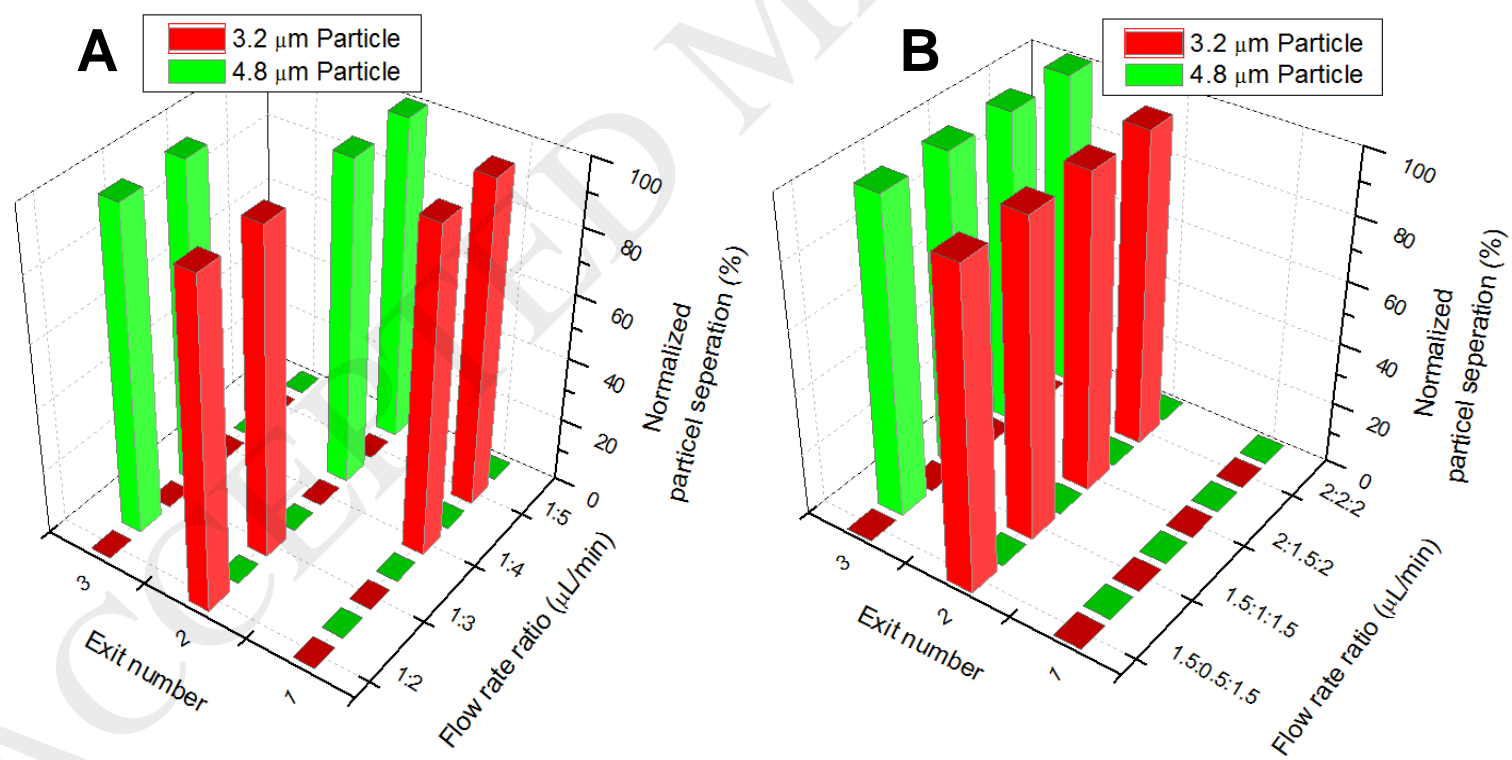

Fig. 9 Simulation results for the separation of two diamagnetic particle types by negative magnetophoresis for (A) two-stream configuration with an optimum sample flow of $1 \mu \mathrm{L} / \mathrm{min}$, and (B) three-stream configuration with an optimum sample/core flow of $0.5-2 \mu \mathrm{L} / \mathrm{min}$. 
Fig. 9 shows the normalized separation efficiency for the $3.2-\mu \mathrm{m}$ and $4.8-\mu \mathrm{m}$ particles for a range of flow rate ratio of the two-stream and three-stream configuration. The simulation suggests that a small and focused sample stream with flow rate lower than that of sheath/cladding stream is more efficient for particle separation. For the two-stream configuration (Fig. 9A), the 3.2- $\mu \mathrm{m}$ particles tend to pass through outlets 1 and 2 . However, the $4.8-\mu \mathrm{m}$ particles mostly pass through outlets 2 and 3 . The total flow rate between $3-4$ $\mu \mathrm{L} / \mathrm{min}$ shows the same behaviour. This behaviour changes with a total flow rate between $5-6$ $\mu \mathrm{L} / \mathrm{min}$. The three-stream configuration (Fig. 9B) offers a more stable separation performance for different flow rate ratios. The optimal separation performance is achieved by focusing the particle between the cladding streams. Most of the 3.2- $\mu \mathrm{m}$ particles going through outlet 2, whereas the 4.2- $\mu \mathrm{m}$ particles pass through outlet 3. Supplementary Figures S5, S6 present the numerical separation performance for the two and three-stream configuration with all possible flow rate ratios.
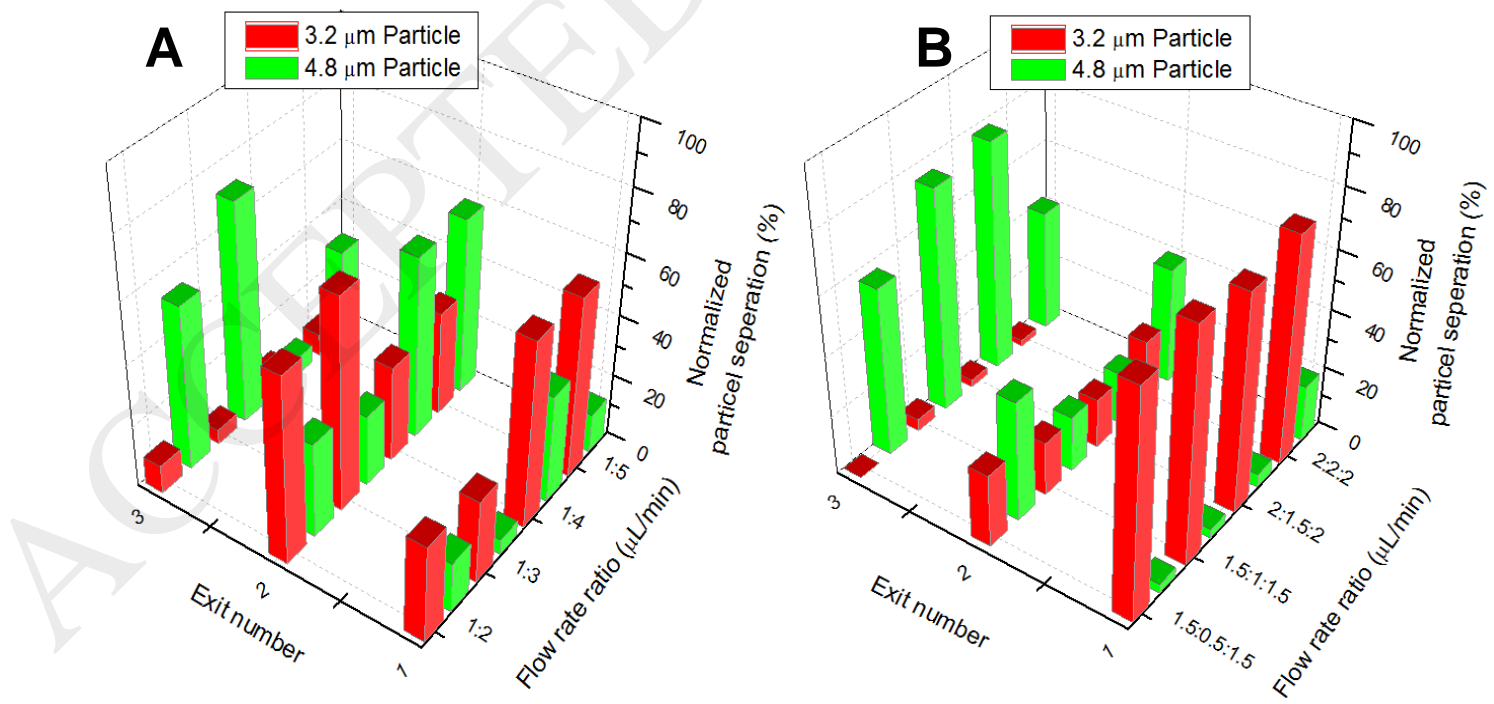

Fig. 10 Experimental results for the separation of two diamagnetic particle types by negative magnetophoresis for the (A) two-stream configuration with a core flow of $1 \mu \mathrm{L} / \mathrm{min}$, and (B) three-stream configuration with a sample/core flow between $0.5-2 \mu \mathrm{L} / \mathrm{min}$. 
Fig. 10A shows the normalized separation efficiency of $3.2-\mu \mathrm{m}$ and $4.8-\mu \mathrm{m}$ particles for different flow rate ratios. The sample to sheath ratio of 1:2 shows a maximum of $60 \%$, and $53 \%$ of separation for the $3.2-\mu \mathrm{m}$ and $4.8-\mu \mathrm{m}$ of particles respectively. The separation performance increases to $68 \%$, and $72 \%$ respectively at the flow rate ratio of $1: 3$. The improved performance is caused by the high sheath flow rate that focuses the sample to a narrow stream. The 3.2- $\mu \mathrm{m}$ particles tend to exit through outlet 2 , whereas, the $4.8-\mu \mathrm{m}$ particles pass through outlet 3 .

Figure 11 shows the separation trajectory for the $3.8-\mu \mathrm{m}$ red and $4.8-\mu \mathrm{m}$ green fluorescent particles. Images of the two-stream configuration for the simulation and experiments are depicted in Fig. 11 A-J, while those of three-stream configuration are depicted in Fig. $11 \mathrm{~K}-\mathrm{T}$. Figures 11A, F, and 11K, P shows the focused sample stream at the inlet for both the simulation and experiments of two and three-stream configurations. As predicted by the simulation (Fig. 7), the three-stream configuration maintains the concentration gradient of the ferrofluid through the entire channel length, enabling the separation of smaller particles. In the two-stream configuration, the concentration is almost homogenous at the channel outlet. Experimental data show weaker separation performance for this configuration, Fig. 11 G-J. Fig. 11B shows clear separation of $3.2-\mu \mathrm{m}$ and $4.8-\mu \mathrm{m}$ particles through inlet 2 , and 3 in the simulation. A number of 3.8- $\mu \mathrm{m}$ particles are visible at inlet 1 in Fig. $11 \mathrm{G}$ due to the attachment of nanoparticles in the experiments. At an optimum flow rate ratio of $1: 3 \mu \mathrm{L} / \mathrm{min}$, the simulation data match with the experimental results due to the right force balance condition. 
Above this flow rate ratio, a mismatch between the simulation and experiment can be observed. Three-stream configuration provides a better separation performance as shown in Fig. 11 K-T. The simulation and experimental trajectories are mostly identical for the $4.8-\mu \mathrm{m}$ green particles. A deviation is observed for the $3.2-\mu \mathrm{m}$ red particles that tend to pass mostly through inlet-1. We suspect that the attachment of magnetic nanoparticles with the smaller red particle pulls them towards the magnetic flux maxima. However, the overall better separation performance is visible throughout the three-stream configuration.






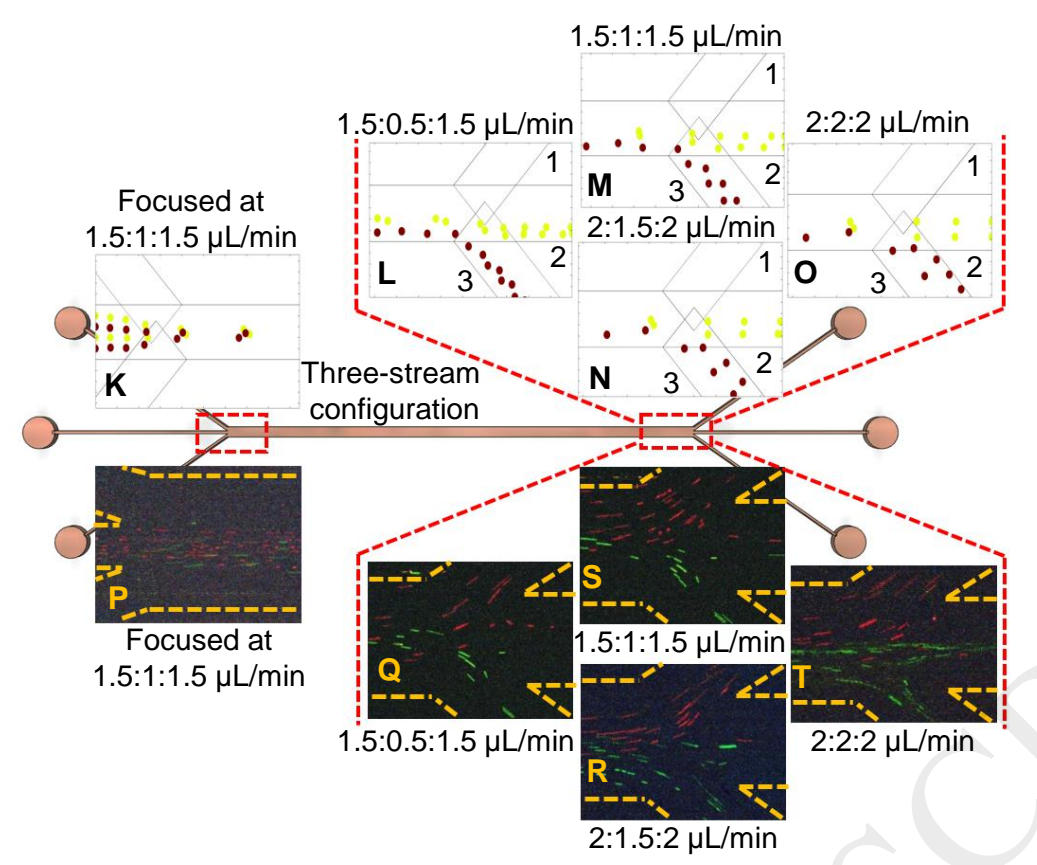

Fig. 11 Experimental trajectories of the 3.8- $\mu \mathrm{m}$ red and 4.8- $\mu \mathrm{m}$ green fluorescent particles for the twostream configuration (A-J), and three-stream configuration (K-T). The multi-stream ferrofluid flow promoted negative magnetophoresis to separate the diamagnetic particle of subtle size difference. The inserts compare the separation performance of the two configurations. 


\section{Accepted Manuscript}

Title: Magnetophoretic separation of diamagnetic particles through parallel ferrofluid streams

Authors: Ahmed Munaz, Muhammad J.A. Shiddiky, Nam-Trung Nguyen

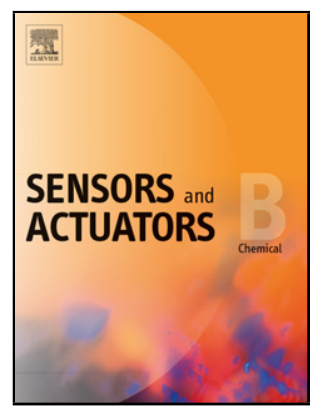

PII:

S0925-4005(18)31424-2

DOI: https://doi.org/10.1016/j.snb.2018.07.176

Reference: SNB 25145

To appear in: Sensors and Actuators B

Received date: 21-3-2018

Revised date: 29-7-2018

Accepted date:

$31-7-2018$

Please cite this article as: Munaz A, Shiddiky MJA, Nguyen N-Trung, Magnetophoretic separation of diamagnetic particles through parallel ferrofluid streams, Sensors and amp; Actuators: B. Chemical (2018), https://doi.org/10.1016/j.snb.2018.07.176

This is a PDF file of an unedited manuscript that has been accepted for publication. As a service to our customers we are providing this early version of the manuscript. The manuscript will undergo copyediting, typesetting, and review of the resulting proof before it is published in its final form. Please note that during the production process errors may be discovered which could affect the content, and all legal disclaimers that apply to the journal pertain. 